\title{
A Novel Power Controlled Sensing Information Gathering for Cooperative Sensing on Shared Spectrum with Primary Spectrum
}

\author{
Mai OHTA $\dagger$, Takeo FUJII $\dagger$, Kazushi MURAOKA $\ddagger$ and Masayuki ARIYOSHI $\ddagger$ \\ $\dagger$ Advanced Wireless Communication Research Center (AWCC), The University of Electro-Communications, \\ 1-5-1, Chofugaoka, Chofu-shi, Tokyo 182-8585 Japan \\ E-mail: \{my, fujii\}@awcc.uec.ac.jp \\ $\ddagger$ System Platforms Research Laboratories, NEC Corporation, \\ 1753, Shimonumabe, Nakahara-ku, Kawasaki, Kanagawa, 211-8666 Japan
}

\begin{abstract}
This paper proposes a novel power controlled sensing information gathering for cooperative sensing on shared spectrum with primary system while jointly using individual sensing results at each node. We apply simultaneous sensing information gathering by using an orthogonal frequency signal. This method can keep the interference power to the primary receiver low. Simulation results show that the power controlled cooperative sensing can obtain good sensing performance without harmful interference toward the primary system.
\end{abstract}

\section{INTRODUCTION}

Dynamic Spectrum Access (DSA) of cognitive radio (CR) technologies can share vacant spectrum of a primary system with a secondary system [1]-[4]. Energy detection is a sensing method checking the received power with a simple implementation, and can successfully detect vacant spectrum even if the structure of the primary signal is unknown. However, due to the wireless channel locality caused by fading and shadowing, the sensing performance degrades. In order to reduce the effect of such kind of wireless channel locality, the cooperative sensing techniques have been studied by many researchers [5]-[7]. The cooperative sensing uses sensing information from surrounding secondary sensing nodes for primary signal detection.

In order to consider a realistic cooperative sensing, a method to gather the cooperative sensing information is very important. We have proposed high efficient information gathering method called orthogonal frequency based sensing information gathering method (OF-SIG) in [8]. Because this method maps the sensing result of each node to the frequency of tone signal, the giving interference is low. This method can also gather the sensing information simultaneously from surrounding sensing nodes. Therefore, our method has potential to share the spectrum with a primary system for gathering the sensing information from surrounding sensing nodes. However, the sensing nodes cannot safely transmit the sensing information without appropriate transmit power design. The sensing nodes would utilize larger transmit power in the spectrum shared channel. Therefore, this paper proposes a novel cooperative sensing method with transmit power control based on local individual sensing of each node. We design the appropriate transmit power of the sensing node while keeping the detection performance without harmful interference.

The proposed transmit power design considers the cooperation probability based on the sensing result in each sensing node and aggregated interference is considered. By using this design, the proposed cooperative sensing method can share the spectrum with the primary system. As a result, this cooperative sensing with OF-SIG can achieve good primary detection performance without the harmful interference, even if on the same spectrum of the primary system.

\section{Cooperative Sensing With Energy Detection}

In this paper, we use an energy detection for spectrum sensing method because it is a simple implementation and has adequate performance [9]. The energy detection method detects unknown signals embedded by comparing the observed received signal power level with a threshold. The threshold is decided based on the probability of false alarm, which is the signal detection probability when the primary user is not existent.

The energy detector of the master node decides the presence of unknown signals comparing two hypotheses, $H_{0}$ and $H_{1}$. These detection statistics in the cooperative sensing can be written as [9]:

$$
\begin{aligned}
& H_{0}: x=\frac{1}{N I} \sum_{i=0}^{I-1} \sum_{n=0}^{N_{i}-1}\left|w_{i}[n]\right|^{2} \\
& H_{1}: x=\frac{1}{N I} \sum_{i=0}^{I-1} \sum_{n=0}^{N_{i}-1}\left|A_{i} s_{i}[n]+w_{i}[n]\right|^{2},
\end{aligned}
$$

where $i$ is the node index number, $I$ is the number of all sensing nodes and $x$ is the test statistic of the cooperative sensing. $w_{i}[n]$ denotes the noise of the $n$th sample, $N$ is the number of all samples. The amplitude of the received signal is $A_{i} s_{i}[n]$ when the transmitter transmits the signal $s_{i}[n]$ and the channel gain is $A_{i}$. 


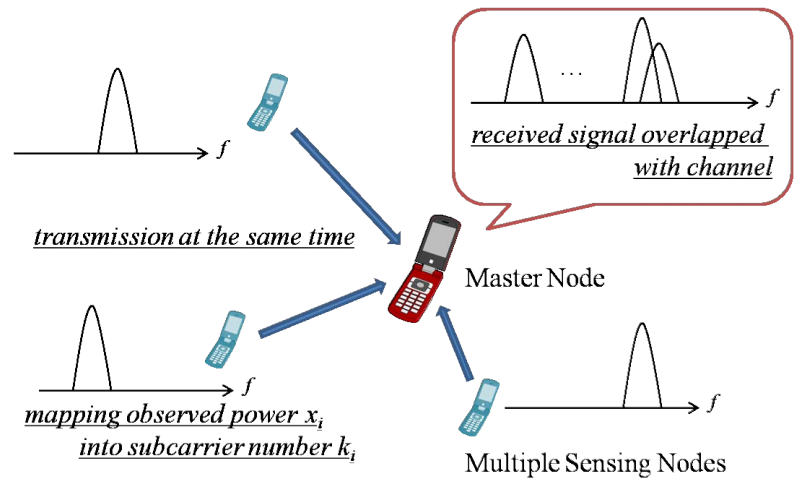

Fig. 1. Simultaneous sensing information transmission using OF-SIG.

The detection function of the presence of the primary user at the master node can be written as:

$$
x>\gamma \text {. }
$$

When Eq. (2) is true, the primary user is judged to be present. This threshold $\gamma$ is decided to satisfy the certain probability of false alarm, $p_{f a}$, if we assume the summed signal is regarded as Gaussian as shown in [7]. Then the threshold $\gamma$ is given by:

$$
p_{f a}=\int p\left(x>\gamma \mid H_{0}\right) d x .
$$

Here, the false alarm probability, $p_{f a}$, denotes that the master node detects the primary signals, nevertheless the primary user is not existence.

\section{Procedure of Sensing Information GATHERING}

For the cooperative sensing, when the sensing nodes transmit the sensing information, a communication channel for sensing information is required. In general, conventional researches consider the dedicated channel for sensing information. However, it reduces the frequency utilization efficiency. Therefore, in this study, we propose a novel power controlled sensing information gathering for cooperative sensing. This paper considers the sensing information on the same spectrum of the primary system without causing the large interference to the primary receiver.

We have proposed OF-SIG as an efficient sensing information gathering method on the primary spectrum. OFSIG transmits tone signal with the decided frequency band according to the sensing result of each sensing node for gathering the sensing information [8]. In [8], we only show the performance of sensing results without considering the aggregated interference at the primary receiver. In general, interference caused by single signal is calculated when the one sensing node is on the nearest location within the secondary communication area. In the worst case like this, it is assumed that information gathering by using the transmit power controlled by this calculation can protect the primary receiver. However, in OF-SIG, the sensing information signals may become harmful interference toward the primary system, because multiple sensing nodes simultaneously transmit the signals. In particular, sensing nodes near the primary receiver

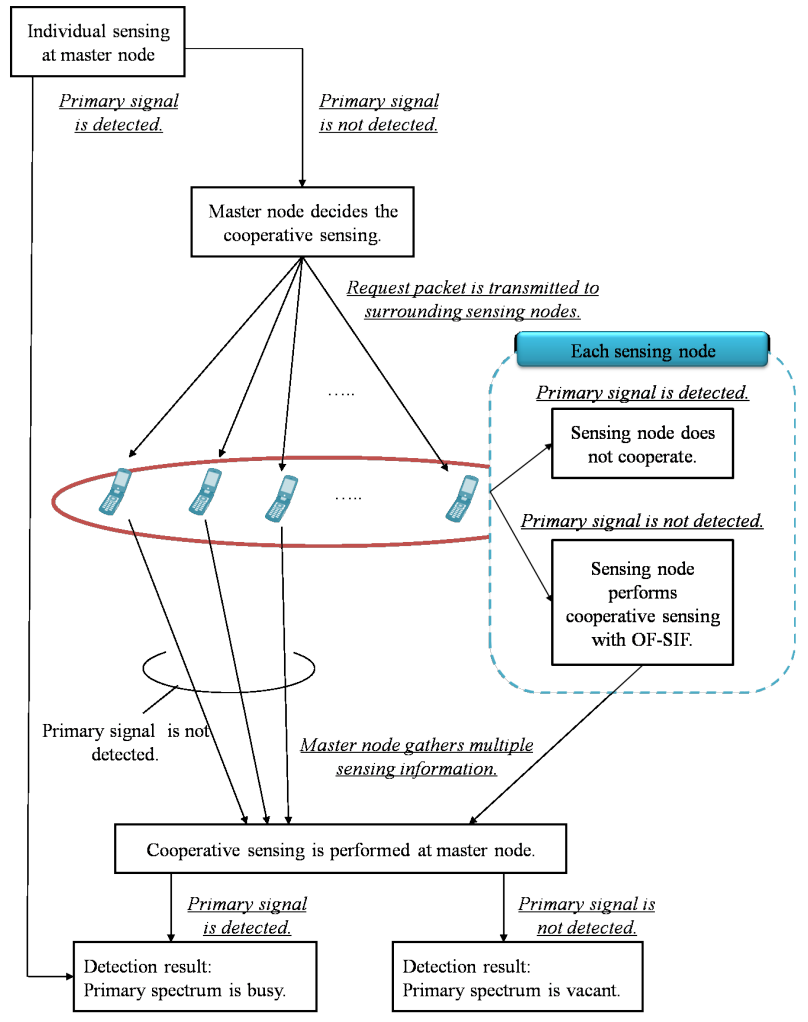

Fig. 2. Diagram of procedures of cooperative sensing with OF-SIG.

should not transmit the sensing information. Therefore, we must decide whether the sensing information can be transmitted or not. Then we consider installing an individual sensing at each sensing node and setting the appropriate power to transmit the sensing information for the cooperative sensing if the primary is not detected at the individual sensing.

For this calculation of the appropriate transmit power, we assume the secondary system already have some parameters. Given parameters are the transmit power of the primary transmitter, the radius of primary service area and the allowable interference power of the primary system. The secondary system can avoid the interference by using these parameters without the knowledge of location of the primary transmitters and receivers. In the proposed cooperative sensing, each sensing node decides whether they can transmit the sensing information or not by using the individual sensing result. Then the master node performs the cooperative sensing by using the received sensing information signal.

In this section, we explain a procedure of the proposed sensing information gathering by using OF-SIG without harmful interference toward the primary receiver. Figure 1 shows a simultaneous sensing information transmission using OFSIG. In order to avoid harmful interference toward the primary system from multiple sensing nodes, sensing nodes control the transmission according to the result of the individual sensing. The diagram of the procedures is shown in Fig. 2.

1) The master node individually senses the received signals to check the status of the primary system in the band. If the master node detects the existence of the primary system, this spectrum is regarded as occupied by the 
primary system. In this paper, we use the signal detection by using the power detector.

2) If the master node does not detect the primary signal, the master node transmits a request packet toward surrounding nodes for gathering sensing information.

3) The nodes which have received the request packet check their own situation by using the individual sensing. From this result, the secondary node near the primary system can avoid the harmful interference toward the primary receiver by withdrawing transmission of the sensing information.

4) The sensing node, which decides to transmit the sensing information, maps the sensing result into the orthogonal frequency signal.

The mapped function is given by [8]:

$$
k_{i}=\left\lfloor x_{i}^{\prime} \frac{N_{c}}{\alpha}\right\rfloor,
$$

where $k_{i}$ denotes the mapped orthogonal frequency like OFDM subcarrier number. $x_{i}^{\prime}$ means the received power at each sensing node normalized by the noise level of the node. $N_{c}$ is the number of orthogonal information bins as $N_{c}$ subcarrier OFDM signal and $\alpha$ denotes the parameter deciding the mapped subcarrier width in the information transmitting bandwidth.

5) All selected sensing nodes simultaneously transmit the orthogonal tone signals with limiting the transmit power which can be decided according to the method shown in Sect. IV.

6) The master node receives the transmitted signals from multiple sensing nodes at the same time and the orthogonal signals are detected in each subcarrier bin. The detection test of each frequency band is given by:

$$
P_{k}>\gamma_{d},
$$

where $P_{k}$ is the received power of $k$ th orthogonal frequency.

When Eq. (5) is true, the master node decides that some primary user sends the sensing information related to this orthogonal frequency. Then the master node reconverts the subcarrier signals to the original sensing information as shown in the following equation:

$$
\hat{x_{j}}=(k+0.5) \frac{\alpha}{N_{c}},
$$

where $\hat{x}_{j}$ denotes the value of the reconverted information mapped from the subcarrier number of orthogonal frequency signals, $j$ is the sensing node index. After that, the cooperative sensing is performed.

From above procedure, the primary signal detection is performed with the cooperative sensing combined with the individual sensing result.

\section{Transmit Power Design for Cooperative SENSING BASED ON AGgREgATED INTERFERENCE}

This section shows the transmit power design which is possible to protect the primary system from the sensing information signals using the primary spectrum.

For simplicity, this section analytically derives the allowable transmit power level by considering our cooperative sensing based on the individual sensing. Figure 3 shows the location of the primary transmitter and receiver and secondary master node. The primary receiver on the boundary of the primary service area should achieve a desired SIR. The averaged interference power, $I_{a}$, is given by:

$$
\begin{gathered}
I_{a}=\int_{0}^{2 \pi} \int_{0}^{d_{c}} P_{m} \rho_{s}(r, \theta) r\left(\frac{\lambda}{4 \pi d_{0}}\right)^{2}\left(\frac{d_{0}}{z}\right)^{n_{p l}} p_{c}(r, \theta) d r d \theta, \\
z=\sqrt{\left(d_{m}-d_{p}\right)^{2}+2 r\left(d_{m}-d_{p}\right) \cos \theta+r^{2}} .
\end{gathered}
$$

Here, $n_{p l}$ is path loss factor, $P_{s}$ denotes the allowable transmit power, $\lambda$ is the wave length, $d_{0}$ is reference distance, $p_{c}($.$) is$ the cooperation probability. $d_{c}$ is the radius of the cooperative sensing area depending on the allowable transmit power of the sensing node, $P_{m}$, in OF-SIG. $d_{m}$ is the distance between the master node and the primary receiver, $d_{p}$ is the radius of the primary service area. $z$ is the distance between the primary receiver and the master node. The number of cooperative sensing nodes is decided by transmission probability of the sensing information at each cooperative sensing node. $d_{c}$ and the cooperative sensing node density $\rho_{s}(r, \theta)$ are given by:

$$
\begin{gathered}
d_{c}=d_{0} \sqrt[n]{\frac{P_{m}}{P_{r}}\left(\frac{\lambda}{4 \pi d_{0}}\right)^{2}}, \\
\rho_{s}(r, \theta)=\rho_{0} p_{c}(r, \theta)=\rho_{0}\left(1-p_{n c}(r, \theta)\right) .
\end{gathered}
$$

Here, $P_{r}$ is the sensing information received power at the master node and is derived from the sensing SNR, $\gamma_{m}$, and $\rho_{0}$ is the density of the sensing nodes.

The cooperation probability decided by individual sensing result of each sensing node, $p_{c}($.$) , is shown by:$

$$
p_{c}(r, \theta)=1-p_{n c}(r, \theta)
$$

$p_{n c}$ is derived primary SNR at the sensing node, $\gamma_{p}\left(z_{i}\right)$. Erfc and $\operatorname{Erfc} c^{-1}$ shows the complementary error function and the inverse of the complementary error function. $N_{i}$ is the number of samples and $p_{f n c}$ denotes false no cooperation probability. $p_{f n c}$ means false transmitting the sensing information as a result of individual sensing, when the primary signal does not exist on the channel. $p_{f n c}$ is decided by the sensing function of the secondary system, because the detection threshold for individual sensing derives signal detection function. Because we consider the worst case, flat Rayleigh fading channel should be considered. Then the probability of no cooperation $p_{n c}$ is given by [10]:

$$
p_{n c}(r, \theta)=\int_{0}^{\infty} \frac{1}{2} \operatorname{Erfc}\left(\frac{\operatorname{Erfc}^{-1}\left(2 p_{f n c}\right)-\sqrt{\frac{N_{i}}{2}} \gamma_{p}(r, \theta)}{\sqrt{2 \gamma_{p}(r, \theta)+1}}\right)
$$




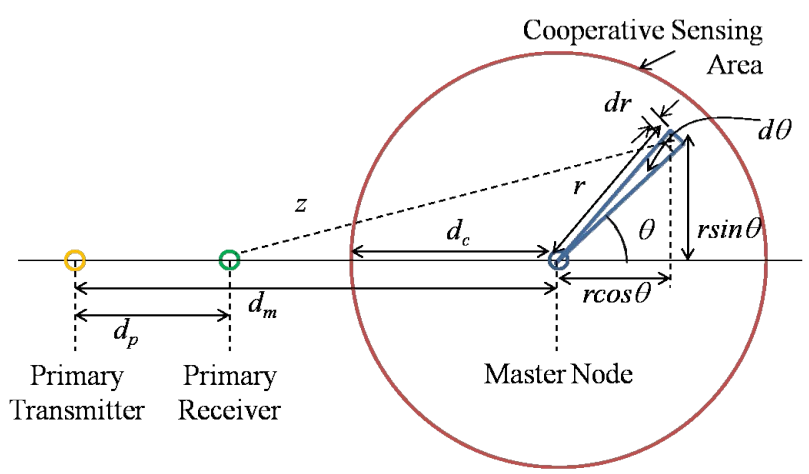

Fig. 3. Primary transmitter and receiver and master node location.

TABLE I

Simulation Conditions.

\begin{tabular}{|l|l|}
\hline Channel model & Rayleigh fading \\
\hline Primary system & 512 subcarrier OFDM \\
\hline Required SIR of primary receiver & $25.0[\mathrm{~dB}]$ \\
\hline Primary transmit power $P_{p}$ & $25.0[\mathrm{dBm} / 512$ subcarriers] \\
\hline Radius of primary service area $d_{p}$ & $100[\mathrm{~m}]$ \\
\hline Noise level & $-90.0[\mathrm{dBm} / 512$ subcarriers] \\
\hline $\begin{array}{l}\text { Sensing information transmission } \\
\text { method }\end{array}$ & $\begin{array}{l}\text { The same frequency based } \\
\text { signal space with primary } \\
512 \text { subcarrier OFDM }\end{array}$ \\
\hline Frequency & $2.4[\mathrm{GHz}]$ \\
\hline Path loss model & $\begin{array}{l}\text { Exponential decay model } \\
\text { (Path loss factor 3, reference }\end{array}$ \\
\hline Probability of false alarm $p_{f a}$ & 0.1 \\
\hline $\begin{array}{l}\text { Probability of false no coopera- } \\
\text { tion } p_{f n c}\end{array}$ & 0.01 \\
\hline Density of sensing nodes $\rho_{0}$ & $0.005\left[\right.$ nodes $\left./ \mathrm{m}^{2}\right]$ \\
\hline Subcarrier detection threshold $\gamma_{d}$ & 11.5 \\
\hline SNR at master node $\gamma_{m}$ & $30[\mathrm{~dB}]$ \\
\hline Mapping parameter $\alpha$ & 2 \\
\hline Number of samples per node & 512 \\
\hline
\end{tabular}

In this paper, in order to design the allowable transmit power under the worst condition, we consider the flat Rayleigh fading and use $p_{n c}$ as shown in Eq. (12). From these equations, we can decide the transmit power of sensing node, $P_{m}$, with keeping $I_{a}$ at the primary receiver by using Eq. (7).

\section{Analytical Evaluation and Simulation Results}

The system parameters used in the analysis and the simulation are shown in Table I. We simulate the performance by changing the distance between the master node and the primary transmitter $d_{m}$. In this paper, primary system information is assumed to be announce from the external server, which gives parameters of the primary system to the secondary receiver nodes. However the location of the primary receivers and transmitter is not required to be known at the secondary system. The secondary system knows the existence of the primary system. The sensing nodes within the area achieve SNR at the master node more than certain SNR requires to cooperate the sensing information.

Figure 4 shows the result of the allowable sensing node transmit power to keep the primary $\mathrm{SIR}=25 \mathrm{~dB}$ which is

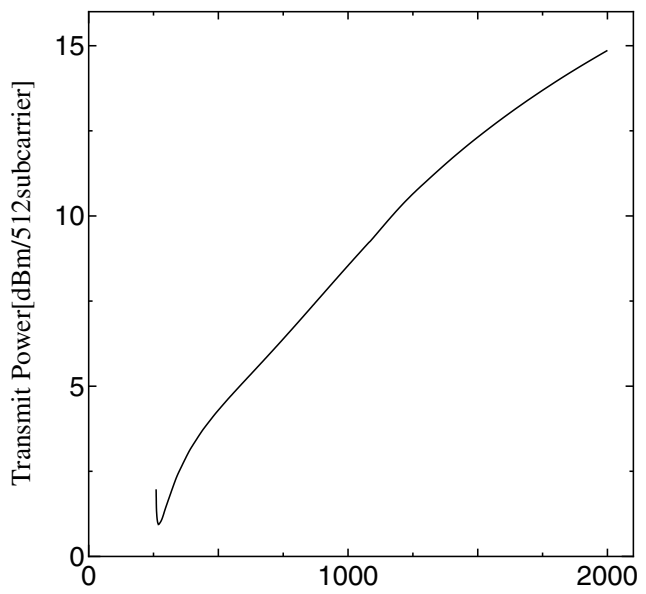

Distance between Primary Transmitter and Master Node[m]

Fig. 4. Secondary transmit power to keep $\mathrm{SIR}=25 \mathrm{~dB}$ at primary receiver.

derived from Eq. (7). From this figure, the minimum allowable secondary transmit power to avoid the interference is decided as $0.94 \mathrm{dBm} / 512$ subcarriers. This is worst location results of the primary transmitter and the receiver. By using this transmit power, the sensing node can protect the primary receiver from the harmful interference even if the secondary nodes cannot know the primary location.

This figure shows that the sensing node near the primary transmitter can utilize large power. This is because the number of cooperation nodes is almost zero by the result of individual sensing result. That is to say, in this case, the probability of cooperation at these nodes, $p_{c}$, is very low, because almost all nodes detect the primary signal. In this location, because the sensing node can certainly receive the signal with the individual sensing, the sensing information is not transmitted. On the other hand, if the secondary nodes are located far away from the primary transmitter, almost all nodes can transmit the information without causing harmful interference due to path loss. The relationship between the number of the cooperative sensing nodes and the location of sensing node is shown in Fig. 5.

From the minimum allowable transmit power of secondary sensing node as $0.94 \mathrm{dBm} / 512$ subcarriers. The radius of the cooperative sensing area is calculated as $39.77 \mathrm{~m}$, the number of average cooperative nodes is calculated as 24 nodes. The exact number of cooperative sensing nodes is decided by including the probability of no cooperation, $p_{n c}$, at each sensing node.

We consider that the false alarm probability combined with both influence of the individual sensing and the cooperative sensing. The each false alarm probability of the individual and the cooperative sensing is $p_{f a}$ and $\left(1-p_{f a}\right) p_{f a}$, respectively. Therefore, combined false alarm probability $p_{f a_{-} c}$ is given by:

$$
p_{f a \_c}=p_{f a}+\left(1-p_{f a}\right) p_{f a} .
$$

In Fig. 6, we compare the performance of only individual sensing and the primary detection with the perfect soft infor- 


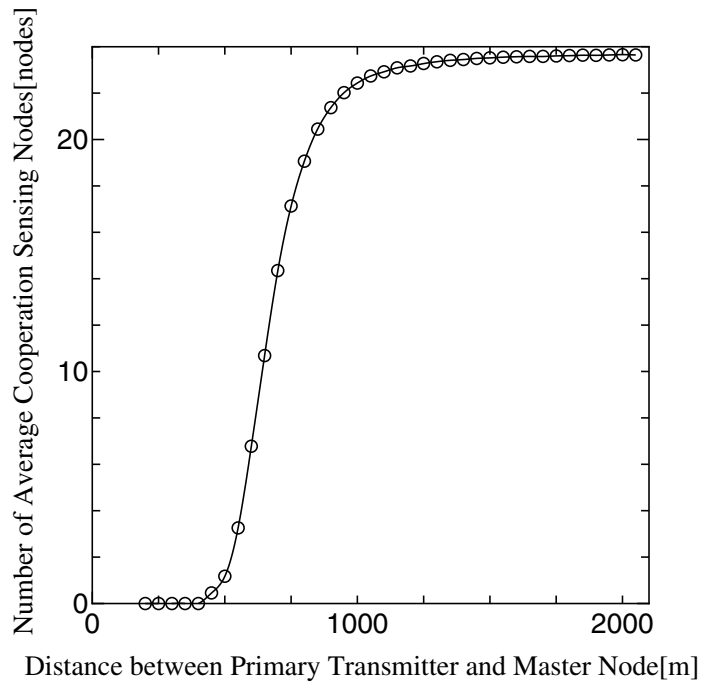

Fig. 5. Number of cooperative sensing nodes.

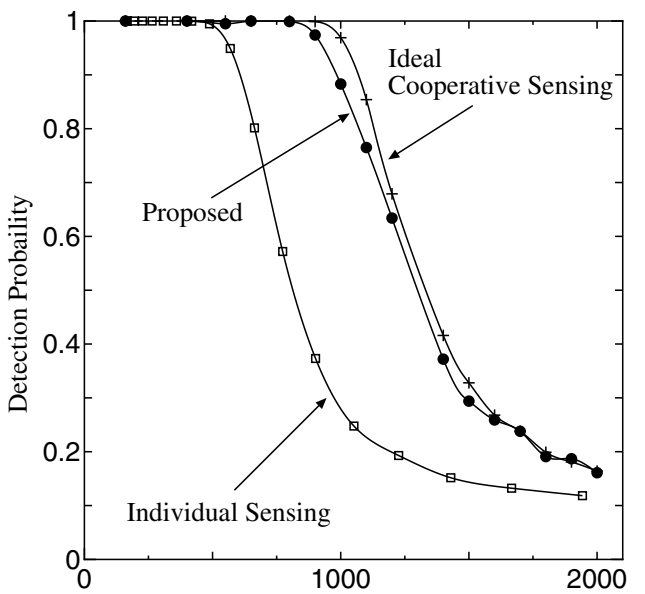

Distance between Primary Transmitter and Master Node[m]

Fig. 6. Comparison of detection performance by simulation.

mation based cooperative sensing. Here, the perfect soft information means that the master node receives the perfect sensing information. From Fig. 6, the performance of the proposed OF-SIG by sharing the spectrum with the primary system is improved compared with the non-cooperative sensing. The performance of the proposed method compared with that of the perfect soft information based cooperative sensing is slightly decreased due to information gathering error with OF-SIG. However, the proposed method can share the channel with the primary system without harmful interference toward the primary system. Moreover, our proposed method donot require the information of the primary location, because we calculate the transmit power considered the worst case.

Figure 7 shows the SIR performance at the primary receiver on the boundary of the primary service area by considering the interference from the sensing nodes for the sensing information transmission with OF-SIG. From this figure, we can confirm that SIR is kept larger than $25 \mathrm{~dB}$ by considering the flat Rayleigh fading. This figure shows the average value so

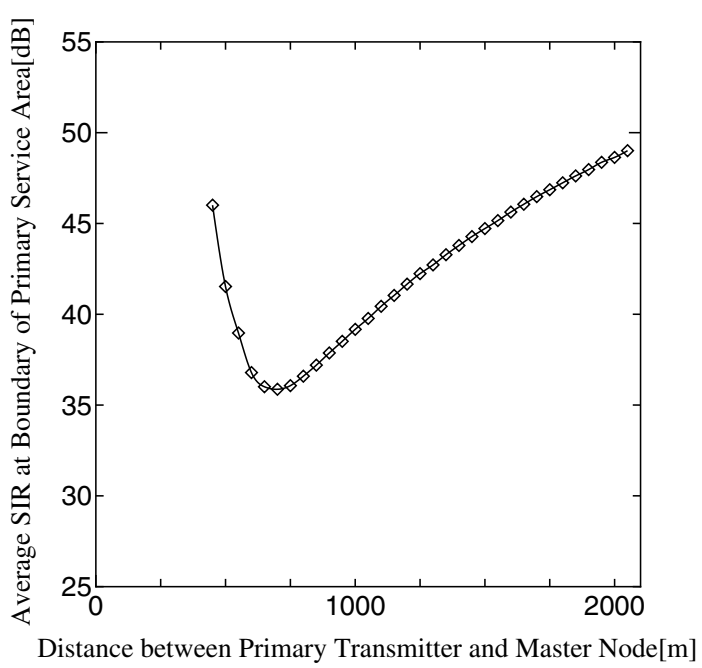

Fig. 7. SIR performance at the worst location primary receiver.

that the SIR is larger then the requirement $\mathrm{SNR}=25 \mathrm{~dB}$.

\section{CONCLUSIONS}

We have proposed a novel power controlled sensing information gathering method based on OF-SIG for cooperative sensing on shared spectrum of the primary system. We analytically design the allowable transmit power of the secondary sensing nodes to share the spectrum with the primary system. We confirm that the primary system is protected from harmful interference by using the proposed transmit power design of sensing nodes with considering the effect of individual sensing.

\section{REFERENCES}

[1] D. Cabric, S. M. Mishra, R. W. Brodersen, "Implementation issues in spectrum sensing for cognitive radios," Proc. ACSSC2004, Nov. 2004.

[2] M. Oner, F. Jondral, "Air interface recognition for a software radio system exploiting cyclostationary," Proc. PIMRC2004, vol.3, pp.1947-1951, Sept. 2004.

[3] Y. Zeng, Y. C. Liang, "Covariance based signal detections for cognitive radio," Proc. DySPAN2007, Dublin, Ireland, April 2007.

[4] H. Urkowitz, "Energy Detection of unknown deterministic signals," Proc. IEEE, vol.55, no.4, pp.523-531, April 1967.

[5] S. M. Mishra, A. Sahai, R. W. Brodersen, "Cooperative sensing among cognitive radios," Proc. ICC2006, pp.1658-1663, 2006.

[6] E. Peh, Y. Liang, "Optimization for cooperative sensing in cognitive radio networks," Proc. WCNC2007, March 2007.

[7] H. Uchiyama, K. Umebayashi, Y. Kamiya, T. Fujii, F. Ono, K. Sakaguchi, "Study on cooperative sensing in cognitive radio based ad-hoc network," Proc. PIMRC2007, Sept. 2007.

[8] M. Ohta, T. Fujii, K. Muraoka, M. Ariyoshi, "A novel method for information gathering by using orthogonal narrowband signal for cooperative sensing in cognitive radio," Proc. IEICE Trans. Commun., vol.E92-B, no.12, Dec. 2009.

[9] J. J. Lehtomaki, M. Juntti, H. Saarnisaari, S. Koivu, "Threshold setting strategies for a quantized total power radiometer," Signal Processing Letters, vol.12, issue 11, pp.796-799, Nov. 2005.

[10] S. Ciftci, M. Torlak, "A comparison of energy detectability models for spectrum sensing," Proc. GLOBECOM, Nov. 2008. 